\title{
Avaliação Psicológica de Obesos Grau III Antes e Depois de Cirurgia Bariátrica
}

\author{
Psychological Evaluation of Grade III Obese Patients \\ Before and After Bariatric Surgery
}

\author{
Jena Hanay Araujo de Oliveira* \& Elisa Medici Pizão Yoshida \\ Pontifícia Universidade Católica de Campinas
}

\begin{abstract}
Resumo
Avalia depressão, ansiedade, sintomas psicopatológicos, alexitimia e estilo defensivo de obesos grau III, antes e depois de cirurgia bariátrica. O estudo seguiu delineamento quase-experimental, de tipo cross-sectional. Contou com grupo pré-cirúrgico $(\mathrm{Gr} 1, N=32)$ e pós-cirúrgico $(\mathrm{Gr} 2, N=33)$, entre seis e 33 meses após a cirurgia. Instrumentos: Inventário de Depressão de Beck (BDI), Inventário de Ansiedade de Beck (BAI), Escala de Avaliação de Sintomas (EAS-40), Versão em Português da Escala de Alexitimia de Toronto (TAS-26) e Versão em Português do Defense Style Questionnaire (DSQ-40). Os dois grupos apresentaram baixos níveis de depressão, ansiedade, sintomas psicopatológicos e alexitimia. No entanto, os níveis do Gr 2 foram significantemente inferiores $(p<0,05)$. Em relação ao estilo defensivo, o Gr1 apresentou mais defesas imaturas $(p<0,02)$ do que o Gr 2.

Palavras-chave: Obesidade severa; mecanismos de defesa; alexitimia; sintomas psicopatológicos; depressão.

Abstract

This study evaluates depression, anxiety, psychopathologic symptoms, alexithymia and defensive style of grade III obese patients, before and after bariatric surgery. The study followed a cross-sectional design, with a group of surgical candidates $(\mathrm{Gr} 1, N=32)$ and one of postoperative patients $(\mathrm{Gr} 2, N=33)$ between 6 and 33 months after the surgery. Instruments: Beck Depression Inventory (BDI), Beck Anxiety Inventory (BAI), Symptom Assessment Scale (EAS-40), Toronto Alexithymia Scale (TAS-26) and Defensive Style Questionnaire (DSQ40). The results pointed to low levels of depression, anxiety, psychopathological symptoms and alexithymia in both groups, although those of Gr2 were significantly lower than those of $\mathrm{Gr} 1(p<0.05)$. Concerning the defensive style, Gr1 showed more immature defense $(p<0.02)$ than Gr2.

Keywords: Severe obesity; defense mechanisms; alexithymia; psychopathological symptoms; depression.
\end{abstract}

A revisão da literatura mostra que boa parte dos trabalhos desenvolvidos com obesos grau III (Índice de Massa Corpórea - IMC $\geq 40 \mathrm{~kg} / \mathrm{m}^{2}$ ) tem como foco a cirurgia bariátrica, que reduz consideravelmente o peso dos pacientes em pouco tempo e diminui comorbidades, tais como: problemas cardiovasculares, diabetes tipo II, hipertensão arterial, distúrbios do sono, tipos de câncer, entre outras (Dixon, Dixon, \& O’Brien, 2003; Garrido Jr., 2001; Geloneze \& Pareja, 2006). A Organização Mundial da Saúde anunciou a nova estimativa de sobrepeso e obesidade no mundo, aproximadamente 1,7 bilhões de pessoas (Deitel, 2003). No Brasil observa-se que nos últimos 20 anos, em todas as faixas etárias, houve um aumento da prevalência da obesidade (Monteiro \& Conde, 2000). Estudos prospectivos mostram que, em 2025, o Brasil será o quinto país do mundo a apresentar problemas de obesidade (Romero \& Zanesco, 2006). Também é possível observar o aumento crescente da obesidade na infância e na adolescência. Como

* Endereço para correspondência: Renascença Medical Center, Av. Colares Moreira, 555, sala 203, Renascença, São Luis, MA, Brasil, CEP 65075-441. E-mail: jenahanay@yahoo.com é o caso dos Estados Unidos onde o número de crianças obesas dobrou e o de adolescentes obesos triplicou desde 1980 (Organização Pan-Americana da Saúde, 2003). A obesidade em crianças e adolescentes, no Brasil, triplicou nos últimos 30 anos (Viuniski, 2003).

Do ponto de vista da psicologia, os trabalhos relacionados à obesidade são relativamente escassos e seus resultados nem sempre consensuais, apesar de haver um reconhecimento de que diferentes fatores psicológicos possam estar associados a ela. Especificamente em relação à obesidade de grau III, enquanto alguns sugerem a existência de comprometimento psicológico significante dentre candidatos à cirurgia bariátrica (Capitão \& Tello, 2002; Dixon et al., 2003; Fabricatore, Wadden, Sarwer, \& Faith, 2005; Mamplekou, Komesidou, Bissias, Papakonstantinou, \& Melissas, 2005), outros indicam que eles estariam mais próximos da população geral, sem níveis elevados de sintomatologia psicopatológica (Dymek, Grange, Neven, \& Alverdy, 2002; Franques \& Ascencio, 2006; Malone \& Mayer-Alger, 2004). Em relação aos indivíduos já submetidos à cirurgia, apesar de também não haver unanimidade, a maioria dos estudos indica uma melhora no 
quadro emocional, principalmente nos primeiros meses, se estendendo até por dois anos (Dixon et al., 2003; Dymek et al., 2002; Malone \& Mayer-Alger, 2004; Mello, 2001; Peixoto \& Geloneze, 2006). Dentre os aspectos psicopatológicos relacionados à obesidade mais pesquisados, encontram-se: a depressão, a ansiedade (Guisado et al., 2002; Matos et al., 2002; Oliveira, Linardi, \& Azevedo, 2004; Vasques, Martins, \& Azevedo, 2004), o transtorno da imagem corporal (Vasques et al., 2004) e os transtornos do comportamento alimentar (Borges, 1998; Matos et al., 2002).

Em relação aos índices de depressão e ansiedade em paciente com obesidade grau III, por exemplo, Franques e Ascencio (2006) encontraram níveis reduzidos de depressão, enquanto que uma pesquisa australiana (Dixon et al., 2003) mostrou que a perda de peso foi associada com uma significante e sustentada queda nos escores de depressão. Um estudo americano (Dymek et al., 2002) que avaliou a qualidade de vida (incluindo a medida de depressão), indicou que antes da cirurgia os pacientes apresentaram um nível leve de depressão e após o procedimento, os escores caíram para depressão mínima. Com relação à ansiedade, a pesquisa de Capitão e Tello (2002) apontou para níveis modestos de ansiedade, utilizando o IDATE. Outras pesquisas apontaram para níveis mais elevados de depressão e ansiedade como, por exemplo, Sánchez et al. (2003) verificaram que do total de 70 participantes $(35 \mathrm{M}$ e $25 \mathrm{H}$ ), $60 \%$ apresentaram desordens do eixo I do DSM- IV, mais freqüentemente a ansiedade e transtorno do humor. $\mathrm{O}$ estudo de Matos et al. (2002) mostrou que 100\% (N=50, $40 \mathrm{M}$ e $10 \mathrm{H}$ ) dos pacientes apresentavam sintomas depressivos antes da cirurgia, sendo que $84 \%$ apresentavam sintomatologia grave. A proporção de indivíduos que exibiam escores indicativos de ansiedade como traço de personalidade foi de $70 \%$ e como estado ansioso foi $54 \%$.

Além dos aspectos sintomatológicos, outras características mais estruturais que informam o modo típico do indivíduo enfrentar as dificuldades, precisam ser melhor investigadas. Dentre estas, destacam-se os mecanismos de defesas, que têm servido como indicativo do modo típico de um indivíduo lidar com os conflitos (Blaya, Kipper, Perez Filho, \& Manfro, 2003) e a alexitimia, relacionada à dificuldade de identificar e/ou descrever o estado emocional (Sifneos, 1973). Os mecanismos de defesa podem ser agrupados de acordo com o grau de maturidade do funcionamento defensivo segundo diferentes níveis: maduro, neurótico e imaturo. O nível maduro envolve o uso de defesas mais adaptadas para controlar o impulso do id, ajustadas adequadamente à realidade e ao mundo externo; imaturo, quando predominam as defesas menos adaptadas e são utilizados alguns recursos para controlar a ansiedade e outros estados afetivos, que podem ser considerados patológicos e o nível neurótico, quando predominam defesas intermediárias entre os funcionamentos maduro e imaturo (Blaya et al., 2003).

Especificamente na área da obesidade, poucos estudos avaliam os mecanismos de defesa. Como exemplo pode-se citar a pesquisa de Ellsworth et al. (1986), que apontou para uma maturidade psicológica maior em sujeitos que mantêm a perda de peso por um longo tempo, e a de Loli (2000), que discute algumas defesas que predominariam em pacientes com obesidade grau III. Entre elas estariam o acting-out, quando os pacientes atuam de forma impulsiva numa tentativa de descarregar as emoções; a projeção, quando delegam o insucesso do tratamento à incompetência do profissional ou até mesmo dos familiares; a negação, quando não reconhecem os aspectos que causam angústia e a somatização, dentre outras. Essas formas de reagir permitem supor que se tratam de pessoas que, possivelmente, apresentam um estilo defensivo imaturo.

Quanto à alexitimia, segundo Yoshida (2006), tem sido dividida em primária ou secundária. A primeira é vista como um traço de personalidade e predispõe à aparição de distúrbios psicossomáticos pela limitação da expressão emocional. A secundária apresenta características semelhantes à primária, mas se manifesta em sujeitos expostos a situações de risco extremo ou que estão ameaçados, inclusive por processo de adoecimento. É um estado transitório ou às vezes prolongado e pode ser visto como uma modalidade adaptativa de tipo "mal menor", para fazer frente a uma situação ameaçadora. Dentre as dificuldades do alexitímico verificam-se, inabilidade para reconhecer as próprias emoções, para distinguir entre sensações corporais e emoções, dificuldade de experienciar as emoções e uma vida de fantasia pobre, que se traduz por uma forma de pensamento concreto (Taylor \& Bagby, 2004). Dentre as pesquisas que avaliaram alexitimia na população com obesidade grau III está a de Adami, Campostano, Ravera, Leggieri e Scopinaro (2001), que apontou um grau de alexitimia similar ao observado em população não-clínica, indicando que ser obeso por si só não influencia a presença desta característica de personalidade. E outros três estudos, no entanto, apontaram sentido contrário, sugerindo a presença de alexitimia na população obesa com transtornos alimentares (Borges, 1998; De Chouly de Lenclave, Florequin, \& Bailly, 2001; Pinaquy, Chabrol, Simon, Louvet, \& Barbe, 2003).

Como consequiência da falta de acordo na área, muitas questões permanecem em aberto, como por exemplo, se existem de fato complicações psicológicas e/ou psicopatológicas significantes associadas à obesidade. Em caso afirmativo, a perda de peso seria acompanhada de melhora da sintomatologia psicopatológica? Ou ainda, será que a perda e manutenção do peso estão relacionadas a um funcionamento psicológico mais maduro e adaptativo? Procurando responder a essas questões definiu-se como objetivos para este estudo, avaliar obesos grau III antes e depois de cirurgia bariátrica, quanto a: severidade da sintomatologia psicopatológica, grau de alexitimia, nível de depressão e ansiedade e mecanismos de defesa. E, comparar as duas avaliações de cada medida, estimando a significância das diferenças entre elas. Do ponto de vista metodológico o estudo segue delineamento cross-sectional, um tipo específico de pesquisa quase-experimental (Bordens \& Abbott, 1999). Baseia-se na comparação de dois grupos cujos participantes estejam em estágios diferentes de um processo desenvolvimental ou tenham idades cronológicas 
diferentes. Assume que o interesse do pesquisador é o de investigar mudanças sem que haja necessidade do acompanhamento dos participantes ao longo do tempo (Bordens \& Abbott, 1999).

\section{Método}

\section{Participantes}

A amostra foi constituída por 65 adultos, pacientes atendidos pelo Sistema Único de Saúde (SUS), no serviço de Cirurgia da Obesidade de um Hospital Universitário de uma cidade da Região Nordeste do Brasil. O Grupo 1 (Gr1, $N=32$ ) foi formado por pacientes que esperavam pela cirurgia e o Grupo 2 (Gr2, N=33), por pacientes já operados, entre seis e 33 meses, e que se mantinham em atendimento ambulatorial. O Grupo 1 ficou composto por: $97 \%$ de mulheres, IMC médio 45,30 kg/m² $(D P=5,41)$, peso médio $111,35 \mathrm{~kg}$, faixa etária entre 31 e 40 anos (47\%), casados ou amasiados (44\%), ensino médio (53\%) seguido do ensino fundamental incompleto (19\%). O Grupo 2 ficou formado por $88 \%$ de mulheres, com IMC médio antes da cirurgia de $46,09 \mathrm{~kg} / \mathrm{m}^{2}(D P=5,03)$ e IMC pós-cirúrgico entre 25 a $29,9 \mathrm{~kg} / \mathrm{m}^{2}(M=29, D P=3,55)$. O peso médio do Gr 2 (pós-cirúrgico) foi de 76,49kg, faixa etária entre 31 e 40 anos (60\%), casados ou amasiados (58\%), ensino médio (33\%) e ensino fundamental incompleto (15\%).

\section{Instrumentos}

Escala de Avaliação de Sintomas - 40 (EAS - 40). De auto-relato, foi adaptada e validada por Laloni (2001) a partir da Symptom Check List - 90 - Revised (SCL-90-R) (Derogatis, 1994). É constituída de 40 itens para avaliar sintomas psicopatológicos, distribuídos em quatro dimensões: F1- Psicoticismo - um contínuo entre psicose e depressão com sintomas de hostilidade e idéias paranóides; F2 Obsessividade-Compulsividade - um conjunto de sintomas de pensamentos e ações repetitivos e acompanhados de desconforto nas relações interpessoais; F3 - Somatização compreende sintomas comuns aos transtornos somáticos e somatoformes; F4 - Ansiedade - um conjunto de sintomas desde a ansiedade generalizada até a ansiedade fóbica, dirigida a situações ou objetos. Uma pesquisa com população clínica indicou boa consistência interna em todos os fatores $[$ alfa $=0,77(\mathrm{~F} 1), 0,83(\mathrm{~F} 2), 0,88(\mathrm{~F} 3)$ e 0,86 (F4)] e precisão de teste reteste, entre sete e 15 dias, igual a: 0,78 (F1), 0,64 (F2), 0,40 (F3) e 0,72 (F4) (Laloni, 2001).

Escala de Alexitimia de Toronto - TAS (Taylor, Ryan, \& Bagby, 1985). De auto-avaliação, idealizada para medir o grau de alexitimia segundo quatro fatores: F1- habilidade de identificar e descrever sentimentos e distinguir sentimentos de sensações corporais; F2 - habilidade para fantasiar ou "sonhar acordado"; F3 - preferência por focalizar eventos externos em vez de experiências internas; F4 habilidade para comunicar os sentimentos a outras pessoas. Possui 26 itens, respondidos em escala Likert de cinco pontos, desde discordo inteiramente (1) até concordo plenamente (5). Os escores totais variam entre 26 e 130 e de acordo com estudos realizados com a versão original
(Taylor et al.,1988), quando acima de 74 (incluindo) são interpretados como presença de alexitimia, enquanto abaixo de 62 (incluindo), ausência de alexitimia. E escores, entre 63 e 73, não permitem avaliações conclusivas. As pesquisas com a versão original sugerem boa consistência interna com coeficientes alfas variando entre 0,75 a 0,79 (Taylor et al.,1985). Com a versão brasileira obteve-se valores dentro deste espectro, tanto para a amostra de universitários, para a qual os valores foram respectivamente 0,71 (amostra total, $n=581$ ), 0,72 (amostra feminina, $n=$ 394) e 0, 72 (masculina, $n=187$ ) (Yoshida, 2000), quanto com a amostra clínica, 0,72 (total, $n=294$ ), 0,73 (feminina, $n=181$ ) e 0,71 (masculina, $n=113$ ). A escala também tem demonstrado boa estabilidade temporal em medidas de teste e reteste de uma semana $(r=0,82)$ e cinco semanas $(r=0,75)$ na versão original (Taylor et al., 1985), assim como na versão brasileira, tanto com estudantes universitários ( $r=0,72$, uma semana) (Yoshida, 2000), quanto com população clínica $[r=0,72(\mathrm{~T}), 0,78(\mathrm{~F})$ e $0,71(\mathrm{M})]$ (Yoshida, 2007). Acrescente-se ainda que a TAS tem sido empregada em alguns estudos com obesos (De Chouly de Lenclave et al., 2001; Morosin \& Riva, 1997; Pinaquy et al., 2003; Troisi et al., 2001; Wagner, 2000).

Inventário de Depressão de Beck - BDI (Cunha, 2001). Foi utilizada a versão de 21 itens que avalia a intensidade dos sintomas de depressão segundo quatro níveis: mínimo, leve, moderado e grave. A intensidade dos sintomas é analisada pelo escore total, obtido com a soma dos escores dos itens. A classificação da depressão é a seguinte: 0-11 mínimo; 12-19 leve; 20-35 moderado e 36-63 grave. Os estudos de teste reteste variam entre 0,48 e 0,86 . O BDI tem sido utilizado no Brasil e em outros países em pesquisas com a população obesa (Jiriki-Babb \& Geliebter, 2003; Marcus, 2004; Matos et al., 2002; Pinaquy et al., 2003; Troisi et al., 2001).

Inventário de Ansiedade de Beck - BAI (Cunha, 2001). É uma escala de auto-relato de 21 itens que medem a intensidade dos sintomas de ansiedade. Os itens devem ser avaliados pelo sujeito numa escala de quatro pontos que refletem: 1 - levemente, 2 - não me incomodou muito, 3 moderadamente: foi muito desagradável mas pude suportar e 4 - gravemente: difícil de suportar. Para cada item atribui-se um escore individual que varia de 0 a 3 pontos. $\mathrm{O}$ escore total é feito pela soma total dos escores individuais e pode variar entre 0 e 63. A interpretação da versão em português é a seguinte: nível mínimo, escores de 0 -10; nível leve, escores de 11 - 19; nível moderado, escores de 20 - 30 e nível grave, escores de 31 - 63. Os índices de correlação entre teste e reteste variaram entre 0,53 e 0,56. Como para os demais instrumentos, tem se verificado o uso desse instrumento em pesquisas com obesos (por ex. Jiriki-Babb \& Geliebter, 2003).

Defensive Style Questionnaire - DSQ- 40 (Andrews, Sinh, \& Bond, 1993). A tradução, adaptação e o estudo da validação do conteúdo da escala para o português do Brasil foram feitos por Blaya et al. (2004). É um instrumento que serve para aferir e monitorar a maturidade dos mecanismos defensivos do ego (Blaya et al., 2003), através da 
identificação do estilo característico de como as pessoas lidam com conflitos, consciente ou inconscientemente. É um questionário auto-aplicável de 40 questões que avaliam 20 defesas relacionadas a três estilos ou fatores: F1imaturo (projeção, agressão passiva, acting-out, isolamento, desvalorização, fantasia-autística, negação, deslocamento, dissociação, cisão, racionalização e somatização); F2 maduro (sublimação, humor, antecipação e supressão) e F3 - neurótico (anulação, pseudo-altruísmo, idealização e formação reativa). $\mathrm{O}$ sujeito responde através de escala Likert de nove pontos, na qual 1 indica discordo plenamente e 9 concordo plenamente (Blaya et al., 2004). O DSQ - 40 já foi traduzido e validado em diferentes países e vem sendo utilizado em pesquisas brasileiras (Blaya et al., 2006; Blaya et al., 2003; Kipper et al., 2005; Kipper et al., 2004). Quanto aos índices de fidedignidade o estilo imaturo revelou melhor consistência (alfa $=0,77)$, sendo seguido pelo estilo maduro $($ alfa $=0,68)$ e neurótico $($ alfa $=0,71)$ Blaya (2005). Em prova de teste e reteste de quatro meses ( $n=33$ ), os coeficientes foram: 0,81 (imaturo); 0,68 (maduro) e 0,71 (neurótico) (Blaya, 2005).

\section{Procedimento}

O projeto foi aprovado pelo Comitê de Ética em Pesquisa com Seres Humanos do Hospital Universitário no qual a pesquisa foi realizada. A seleção do Grupo 1 se baseou na lista de inscrição dos pacientes que aguardavam por cirurgia e que apresentavam IMC igual ou superior a $40 \mathrm{Kg} / \mathrm{m}^{2}$. Para o Grupo 2, selecionou-se pacientes que tinham sido submetidos à cirurgia há, no mínimo, seis meses. A aplicação dos instrumentos foi feita nas dependências do hospital, de forma individual e antecedida de uma entrevista em que foram colhidas informações sócio demográficas e o consentimento dos participantes, após a leitura do Termo de Consentimento Livre e Esclarecido.

Os dados foram analisados calculando-se, para cada grupo, os escores médios (totais e parciais, quando se aplicava) e desvios padrão de cada um dos instrumentos utilizados. A seguir, as médias do Grupo 1 e 2 foram comparadas por meio do emprego de testes $t$ para amostras independentes e presumindo-se variâncias equivalentes.

\section{Resultados}

No que respeita à composição dos Grupos 1 e 2, apesar de não ter se dado de forma aleatória, ambos apresentaram perfil sócio-demográfico semelhante, com predomínio de mulheres, casadas ou amasiadas, com idade entre 30 e 40 anos e com nível educacional médio. Apenas no que concerne ao IMC houve diferença estatística significante entre as médias dos grupos, antes e depois da cirurgia ( $t$ para amostras independentes presumindo-se variâncias equivalentes $=14,40$, tc bi-caudal=1,99). A Tabela 1 apresenta os resultados médios de ambos os grupos em cada um dos instrumentos de avaliação utilizados, os respectivos desvios-padrão, escores mínimo e máximo e valores de $t$ para amostras independentes, presumindo-se variâncias equivalentes.
Em relação aos níveis de ansiedade, avaliados pelo BAI, em ambos os Grupos predominaram os níveis de ansiedade mínima e leve, ainda que o Grupo 2 tenha apresentado escore médio significantemente inferior ao do Grupo 1 ( $t=2$, $88, p<0,01)$. Quanto à distribuição dos participantes de acordo com os níveis de ansiedade, no Grupo $153,12 \%$ apresentavam nível mínimo de ansiedade, 31,25\% ansiedade leve, $12,5 \%$ ansiedade moderada e apenas 3,12\% tinham sintomas ansiosos graves. No Grupo 2, os resultados apontaram para 81,81\% com ansiedade mínima, 12,12\% leve, $6,06 \%$ com ansiedade moderada, e nenhum participante apresentou ansiedade grave.

Quanto aos sintomas de depressão, predominaram os níveis mínimos e leve nos dois grupos, apesar do Grupo 2 ter escore médio no BDI significantemente inferior ao Grupo $1(t=4,97, p<0,001)$. No Grupo $1,34,37 \%$ apresentaram depressão considerada mínima, 40,62\% depressão leve, $25 \%$ depressão moderada e nenhum participante com depressão grave. No Grupo 2, 81,81\% apresentaram depressão considerada mínima, 15,15\% leve, 3,03\% moderada e nenhuma grave. As avaliações dos sintomas psicopatológicos com a EAS-40 indicaram que o Grupo 1 apresentou escore médio total mais elevado que o Grupo 2 ( $t=2,97$, $p<0,005)$. As diferenças também foram significantes nos sintomas que dizem respeito ao F2 (obsessividade-compulsividade) e F3 (somatização), respectivamente, $t=2$, $21,(p<0,005)$ e $t=3,42(p<0,001)$, indicando uma diminuição da presença desses sintomas no Grupo 2. Nos Fatores 1 e 4, apesar dos escores do Grupo 1 terem sido em média superiores ao do Grupo 2, as diferenças não foram significantes ( $t=1,11$ e $t=1,97$, respectivamente).

Quanto à medida de severidade dos sintomas psicopatológicos, como não há dados normativos para a EAS-40, a interpretação dos escores deve ser feita comparando-os com os de outras pesquisas brasileiras. Verificou-se que o IGS (Índice Geral de Severidade) médio tanto do Gr1 (0,48 e $D P=0,35)$ quanto do $\operatorname{Gr} 2(0,31$ e $D P=0,20)$ foram menores do que os de duas pesquisas com população clínica, a de Laloni (2001) com pacientes de hospital geral e a de Pregnolatto (2005) com pacientes com insuficiência renal crônica. Na primeira, a média foi de $1,47(D P=0,94)$ no sexo feminino e $1,36(D P=0,92)$ para o sexo masculino. No segundo estudo, a média total foi de 0,63 $(D P=0,37)$. Na pesquisa com universitários (Yoshida \& Silva, 2007), a média do IGS do sexo masculino foi de $0,55(D P=0,40)$ e do sexo feminino, 0,60 $(D P=0,41)$, mais próximas dos valores verificados no presente estudo. $\mathrm{O}$ que se constatou, portanto, foi que a severidade da sintomatologia geral ficou mais próxima da população geral e, portanto, nãoclínica.

Em relação à alexitimia, houve diferença significante entre os Grupos quanto aos escores totais da TAS ( $t=2,78$, $p<0,01$ ). O mesmo ocorreu com o F1 (habilidade de identificar e descrever sentimentos e distinguir sentimentos de sensações corporais), em que o Grupo 1 obteve escore médio superior $(t=3,08, p<0,005)$. Em relação aos demais fatores as diferenças não foram significantes $(p>0,05)$. Como a TAS também não possui normas para a população 
Psicologia: Reflexão e Crítica, 22(1), 12-19.

Tabela 1

Escore Médio, Desvio Padrão, Escores Mínimo e Máximo do BDI, BAI, EAS-40, TAS e DSQ-40 e Teste t dos Grupos 1 e 2

\begin{tabular}{|c|c|c|c|c|c|c|c|}
\hline \multirow[t]{2}{*}{ Instrumentos } & \multirow[b]{2}{*}{$M$} & \multicolumn{2}{|c|}{ Grupo 1} & \multicolumn{3}{|c|}{ Grupo 2} & \multirow[b]{2}{*}{ Teste $t$} \\
\hline & & $D P$ & $\min -\max$ & $M$ & $D P$ & $\min -\max$ & \\
\hline$B A I$ & 11,62 & 7,20 & $1-32$ & 6,90 & 6,09 & $0-22$ & $2,88 *$ \\
\hline$B D I$ & 15,06 & 7,27 & $2-31$ & 7,60 & 4,64 & $0-20$ & $4,97 * * *$ \\
\hline \multicolumn{8}{|l|}{ EAS-40 } \\
\hline total & 0,48 & 0,35 & $0,025-1,05$ & 0,31 & 0,20 & $0,05-0,87$ & $2,97 * * *$ \\
\hline F1(psicoticismo) & 0,29 & 0,23 & $0-0,8$ & 0,22 & 0,26 & $0-1$ & 1,11 \\
\hline F2 (obses.-compulsividade) & 0,61 & 0,39 & $0-1,4$ & 0,42 & 0,28 & $0-1,1$ & $2,21 * * *$ \\
\hline F3 (somatização) & 0,73 & 0,41 & $0,1-1,5$ & 0,42 & 0,31 & $0-1,2$ & $3,42 * * *$ \\
\hline F4 (ansiedade) & 0,29 & 0,26 & $0-0,9$ & 0,17 & 0,20 & $0-0,8$ & 1,97 \\
\hline \multicolumn{8}{|l|}{ TAS } \\
\hline total & 68,25 & 9,81 & $50-86$ & 61,60 & 9,41 & $35-78$ & $2,78 *$ \\
\hline F1(identificar sentimento) & 27,90 & 7,65 & $14-44$ & 22,63 & 6,03 & $13-41$ & $3,08 * * *$ \\
\hline F2 (fantasiar) & 10,68 & 3,40 & $4-15$ & 11,06 & 2,93 & $4-16$ & $-0,47$ \\
\hline F3 (comunicar sentimento) & 16,34 & 2,77 & $9-22$ & 15,51 & 2,38 & $10-22$ & 1,29 \\
\hline F4 (foco no exterior) & 13,31 & 2,72 & $9-12$ & 12,39 & 3,86 & $5-20$ & 1,10 \\
\hline \multicolumn{8}{|l|}{ DSQ } \\
\hline F1- nível imaturo & 8,44 & 2,29 & $5,25-14,25$ & 7,25 & 1,75 & $3,66-10,83$ & $2,38 * *$ \\
\hline F2- nível maduro & 11,76 & 2,50 & $5-16,5$ & 12,26 & 2,27 & $7,5-16,75$ & $-0,86$ \\
\hline F3- nível neurótico & 10,23 & 2,41 & $6-15,5$ & 9,56 & 3,05 & $4-16$ & 0,98 \\
\hline \multicolumn{8}{|l|}{ Defesas Imaturas } \\
\hline Projeção & 3,34 & 2,20 & $1-8,5$ & 1,86 & 1,11 & $1-5$ & $2,96 * * *$ \\
\hline Agressão passiva & 3,65 & 2,33 & $1-9$ & 2,5 & 1,43 & $1-5,5$ & $2,44 * *$ \\
\hline Acting out & 4,87 & 2,40 & $1-9$ & 5 & 2,73 & $1-9$ & $-0,20$ \\
\hline Isolamento & 3,75 & 1,91 & $1-9$ & 3,46 & 2,03 & $1-8$ & 0,60 \\
\hline Desvalorização & 3,95 & 2,08 & $1-8$ & 2,77 & 1,95 & $1-8$ & $2,40 * *$ \\
\hline Fantasia autística & 3,57 & 2,38 & $1-9$ & 2,27 & 1,43 & $1-5,5$ & $2,70 *$ \\
\hline Negação & 4,20 & 2,13 & $1-9$ & 3,93 & 2,28 & $1-9$ & 0,50 \\
\hline Deslocamento & 4,42 & 2,31 & $1-10$ & 2,21 & 1,39 & $1-6$ & $4,80 * * *$ \\
\hline Dissociação & 4,09 & 1,82 & $1-8,5$ & 4,57 & 2,11 & $1-8,5$ & -1 \\
\hline Cisão & 4,57 & 2,81 & $1-16$ & 4,69 & 1,92 & $1-9$ & $-0,20$ \\
\hline Racionalização & 6,54 & 2,22 & $2,5-14$ & 6,45 & 1,98 & $1-9$ & 0,17 \\
\hline Somatização & 4,79 & 2,66 & $1-9$ & 3,95 & 2,19 & $1-9$ & 1,40 \\
\hline \multicolumn{8}{|l|}{ Defesas maduras } \\
\hline Antecipação & 6,92 & 2,05 & $1-9$ & 7,01 & 1,63 & $3-9$ & $-0,20$ \\
\hline Humor & 5,42 & 1,95 & $1-9$ & 6,06 & 1,92 & $1-9$ & $-1,36$ \\
\hline Supressão & 5,37 & 2 & $1-9$ & 5,48 & 1,40 & $3-9$ & $-0,26$ \\
\hline Sublimação & 5,81 & 2,10 & $2-9$ & 5,90 & 2,34 & $2-9$ & $-0,16$ \\
\hline \multicolumn{8}{|l|}{ Defesas neuróticas } \\
\hline Pseudo-altruísmo & 6,23 & 1,69 & $3-9$ & 5,40 & 2,29 & $1-9$ & 1,69 \\
\hline Idealização & 4,10 & 2,19 & $1-8$ & 4,01 & 2,14 & $1-9$ & 0,16 \\
\hline Formação reativa & 5,35 & 2,14 & $1-9$ & 5,54 & 2,40 & $1-9$ & $-0,33$ \\
\hline Anulação & 4,76 & 2,13 & $1-9$ & 4,46 & 2,40 & $1-9$ & 0,53 \\
\hline
\end{tabular}

Nota. Teste $t$ dos Grupos 1 e 2; * $p<0,01 * * p<0,02 * * * p<0,001$.

obesa, os resultados foram analisados utilizando-se como referência os resultados de outras pesquisas brasileiras feitas com essa escala. Comparando-se os escores totais de ambos os grupos (Gr 1: $M=68,25$ e $D P=9,81$ e Gr $2: M=$ $61,60$ e $D P=9,41)$ com os de: dependentes de álcool $(M=$ 78,65 e $D P=8$ ) (Maciel \& Yoshida, 2006); pacientes com Doença de Crohn e retocolite ulcerativa $(M=76,44$ e $D P$ $=10,13$ ) (Guimarães \& Yoshida, 2008); pacientes com in- suficiência renal crônica $(M=74$ e $D P=7,52)$ (Pregnolatto, 2005); portadores de glaucoma $(M=73,18$ e $D P=11,56)$ (Regina, 2005) e pacientes de hospital geral $(M=72,8$ e $D P=9,15$ ) (Yoshida, 2007), constatou-se que ficaram abaixo de todos eles. Por outro lado, os escores médios ficaram mais próximos daqueles encontrados em amostras de universitário por Silva (2004) $(M=67,80, D P=8,85) \mathrm{e}$ Yoshida (2000) $(M=63,13$ e $D P=10,12)$. Tomando como 
pontos de corte para a alexitimia o escore 74 e para não alexitimia, 62, no Grupo 1 28,12\% foram considerados alexitímicos e $31,25 \%$ não-alexitímicos. Enquanto que $40,62 \%$ ficaram entre 63 e 73. Nessa faixa uma avaliação conclusiva sobre a alexitimia não é possível. No Grupo 2 $45,45 \%$ foram considerados não-alexitímicos e apenas $9,09 \%$ alexitímicos. E, 45,45\% ficaram entre 63 e 73 . De acordo com o DSQ-40 (Tabela 1), apenas no estilo defensivo imaturo a diferença entre os grupos foi significante, indicando uma tendência do Gr 1 a apresentar mais defesas imaturas do que o Grupo $2(p<0,02)$. Efetivamente, os escores médios de defesas tais como: projeção $(p<0,005)$, agressão passiva $(p<0,02)$, desvalorização $(p<0,02)$, fantasia autística $(p<0,01)$ e deslocamento $(p<0,001)$, foram significantemente inferiores no Grupo 2.

\section{Discussão}

Em relação às variáveis sócio-demográficas, a diferença do IMC médio do Gr 1 pré-cirúrgico em relação ao Gr 2 pós-cirúrgico se mostrou compatível com a de outros estudos, reforçando as observações de que a cirurgia bariátrica é um tratamento que tem sucesso na maioria dos casos, pois promove o emagrecimento (Dixon et al., 2003; Dymek et al., 2002; Malone \& Mayer-Alger, 2004; Mello, 2001; Peixoto \& Geloneze, 2006). O predomínio de mulheres na amostra também coincide com o que é apontado por pesquisas realizadas em outros contextos sócio-culturais, em que as mulheres correspondem à maioria dos pacientes submetidos à cirurgia bariátrica (Capitão \& Tello, 2002; Fabricatore et al., 2005; Franques \& Ascencio, 2006).

O nível de sintomas depressivos verificado antes da cirurgia era, em média, leve e diminuiu após a cirurgia, corroborando os resultados de outras pesquisas em que níveis semelhantes foram encontrados (Dixon et al., 2003; Dymek et al., 2002; Franques \& Ascencio, 2006). Isto é, os dados sugerem que a obesidade não necessariamente é acompanhada de sintomatologia depressiva severa ou grave. No entanto, deve-se destacar que $25 \%$ dos participantes do Grupo 1 apresentaram nível moderado de sintomas depressivos, sugerindo, portanto, que um quarto da população atendida merece atenção quanto a esse aspecto, e que a população de obesos de grau III é heterogênea no que respeita à severidade da depressão.

Quanto à ansiedade, os resultados apontaram para um nível leve no Grupo 1 e nível mínimo no Grupo 2, apresentando similaridade com o estudo de Capitão e Tello (2002), que encontrou níveis baixos de ansiedade em mulheres obesas, utilizando o IDATE. Procurando interpretar estes dados, propõem-se como hipóteses explicativas que os candidatos à cirurgia já contam com o "recurso de cura" para sua obesidade e não se preocupam muito em perder peso, diferentemente daqueles tratados convencionalmente, que devem fazer dietas, estão sujeitos a restrição alimentar, fatores que podem funcionar como ansiogênicos. Ou seja, a expectativa de que o tratamento cirúrgico possibilite a perda de peso e sua manutenção, em definitivo, pode ser uma variável importante nesse processo. Em relação ao grupo pós-cirúrgico, o sucesso da cirurgia, com a diminuição do peso, explicaria a diferença significante em relação ao Gr 1.

Em relação aos sintomas psicopatológicos medidos com a EAS-40, os resultados são semelhantes aos de outras pesquisas que sugerem que a severidade da psicopatologia presente nos casos de obesidade é semelhante à da população geral (Dymek et al., 2002; Franques \& Ascencio, 2006; Malone \& Mayer-Alger, 2004). Isto é, apesar da obesidade grau III ser freqüentemente acompanhada por várias comorbidades médicas, ela não deve ser vista como necessariamente associada a problemas de ordem psicológica. $\mathrm{O}$ mesmo pode ser dito em relação à alexitimia visto que os dois grupos apresentaram escores médios bem próximos aos de amostras de universitários brasileiros, considerados parte da população não-clínica (Silva, 2004; Yoshida, 2000). Os resultados também foram similares aos de outras pesquisas, tais como a de Adami et al. (2001), de Borges (1998), De Chouly de Lenclave et al. (2001) e de Loli (2000) que indicaram que a obesidade não é condição necessária para o aparecimento de alexitimia. No entanto, procurando compreender a diferença entre os escores médios dos grupos, ou tentando analisar o fato de no Gr 1 haver mais alexitímicos $(28,12 \%)$ do que no Gr $2(9,09 \%)$, cogita-se que estas diferenças se devam à incidência de alexitimia secundária no Gr 1. Conforme referido, a alexitimia secundária surge em situações específicas em que os limites dos recursos adaptativos da pessoa são ultrapassados, ou ainda como um fator de proteção contra a significação emocional da gravidade da doença (Yoshida, 2006). Nesse sentido, em candidatos à cirurgia ela teria função protetora contra o sofrimento emocional associado à obesidade, pois nessa condição os riscos e a gravidade da obesidade também ficam minimizados. Todavia, ainda que existam obesos alexitímicos, é preciso enfatizar que essas interpretações requerem cautela quanto à generalização dos resultados e rotulação destes pacientes.

Em relação aos estilos defensivos, foram observadas diferenças entre os grupos apenas quanto ao nível de defesas imaturas, muito mais freqüentes no grupo Gr 1. Esses dados coincidem com os de Ellsworth et al. (1986) que revelaram que há uma melhora no funcionamento defensivo em direção a um estilo mais maduro, nas pessoas que conseguem perder e manter o peso. Dentre as defesas que tiveram escores reduzidos no $\mathrm{Gr} 2$, de forma significante, quando comparados aos do Gr 1, estão: a projeção, a agressão passiva, a desvalorização, a fantasia autística, o deslocamento e o acting-out. Fato que foi semelhante ao verificado na literatura quanto à presença de algumas defesas específicas nessa população (Ellsworth et al., 1986; Loli, 2000).

Perry (1990) define da seguinte forma a função das defesas que tiveram significância neste estudo: "O acting-out permite ao sujeito descarregar ou exprimir sentimentos e impulsos em vez de tentar tolerá-los ou refletir sobre eventos dolorosos que os estimularam" (p. 5). A projeção permite que o sujeito lide com emoções e motivos que, se admitidos, fariam-no sentir-se envergonhado e humilha- 
do, atribuindo-os a outrem. A agressão-passiva é caracterizada pelo descarregar de sentimentos de hostilidade e ressentimento de uma maneira indireta e dissimulada em relação a outros. No caso da obesidade, um exemplo do uso dessa defesa é a agressividade interna despertada pela raiva que sente em não conseguir emagrecer e que é expressa com certa hostilidade contra si mesmo ou contra os outros. Ou então, o próprio comportamento alimentar sem controle pode refletir agressão a si mesmo. "A desvalorização refere-se ao uso de afirmações desabonadoras, ou outras afirmações negativas sobre alguém ou sobre si mesmo, de maneira a valorizar a auto-estima" (Perry, 1990, p. 30). Essa defesa está relacionada diretamente à auto-estima, que no caso da população obesa tende a ser baixa. "A fantasiaautística permite ao sujeito obter gratificação temporária e substituta por meio do sonhar, uma solução para um problema ou conflito do mundo real" (Perry, 1990, p. 27). Além do que, essa defesa substitui as atividades da vida real por fantasia excessiva, e é característica de um ego mais rígido. Ainda de acordo com Perry (1990), o deslocamento permite a expressão de um afeto, impulso ou ação em direção a uma pessoa ou outro objeto que possua semelhança com o objeto que inicialmente despertou o afeto ou impulso. Um exemplo de uso dessa defesa é quando o problema da obesidade é deslocado para outro alvo de preocupação, de forma a dissipar a angústia que essa condição provoca.

Apesar dos resultados coincidirem com os de outras pesquisas na área é preciso assinalar algumas limitações do estudo que sugerem cautela na generalização dos resultados. A primeira diz respeito à representatividade da amostra, muito pequena se comparada ao número de obesos de grau III na população. Outro fator refere-se à presença predominante de mulheres. E finalmente, uma palavra sobre o delineamento de tipo cross-sectional, que não permite conclusões sobre mudanças propriamente ditas nas variáveis estudadas, havendo necessidade de se cingir apenas à constatação de diferenças entre os grupos pré e pós-cirúrgicos. Tendo em mente estas limitações pode-se, portanto, apenas supor que a perda de peso deve ter contribuído para a redução da sintomatologia psicopatológica e para as diferenças na utilização das defesas verificadas entre os grupos. Por outro lado, não se deve pensar numa característica de personalidade única e nem tampouco adotar uma postura única diante da associação da obesidade com aspectos psicológicos e psicopatológicos. O importante é compreender, em cada caso, quais fatores estão presentes e quais podem propiciar sofrimento emocional e nesse sentido, buscar propostas terapêuticas adequadas.

\section{Referências}

Adami, G. F., Campostano, A., Ravera, G., \& Scopinaro, N. (2001). Alexithymia and body weight in obese patients. Behavioral Medicine, 27(3), 121-123.

Andrews, G., Singh, M., \& Bond, M. (1993). The Defense Style Questionnaire. Journal of Nervous and Mental Disease, 181(4), 246-256.

Blaya, C. (2005). Tradução, adaptação e validação do Defensive Style Questionnaire (DSQ-40) para o português brasileiro. Dissertação de Mestrado não-publicada, Universidade Federal do Rio Grande do Sul, Porto Alegre, RS.
Blaya, C., Dorneles, M., Blaya, R., Kipper, L., Heldt, E., Isolan, L., et al. (2006). Do defense mechanisms vary according to the psychiatric disorder? Revista Brasileira de Psiquiatria, 28(3), 179-183.

Blaya, C., Kipper, L., Heldt, E., Isolan, L., Ceitin, L. H., Bond, M., et al. (2004). Versão em português do Defensive Style Questionnaire (DSQ - 40) para avaliação dos mecanismos de defesa: Um estudo preliminar. Revista Brasileira de Psiquiatria, 26(4), 255-258.

Blaya, C., Kipper, L., Perez, J. B., Filho, \& Manfro, G. G. (2003). Mecanismos de defesa: Uso do Defensive Style Questionnaire. Revista Brasileira de Psicoterapia, 5(1), 67-80.

Bordens, K. S., \& Abbott, B. B. (1999). Research design and methods: A process approach (4. ed.). Chicago: Mayfield.

Borges, M. B. F. (1998). Estudo do transtorno da compulsão alimentar periódica em população de obesos e sua associação com depressão e alexitimia. Dissertação de Mestrado nãopublicada, Universidade Federal de São Paulo, SP.

Capitão, C. G., \& Tello, R. R. (2002). Traço e estado de ansiedade em mulheres obesas [Versão eletrônica]. Revista de Psicologia Hospitalar, 2(2). Retrieved from http://pepsic.bvs-psi.org. br/scielo.php?script=sci_arttext\&pid=S1677740920040002 $00002 \& \operatorname{lng}=$ pt\&nrm=iso

Cunha, J. A. (2001). Manual da versão em português das Escalas Beck. São Paulo, SP: Casa do Psicólogo.

De Chouly de Lenclave, M. B., Florequin, C., \& Bailly, D. (2001). Obesity, alexithimia, psychopathology and binge eating: A comparative study of obese patients and 32 controls. Encephale, 2(4), 343-350.

Deitel, M. (2003). Overweight and obesity worldwide now estimated to involve 1.7 billion people. Obesity Surgery, 13, 329-330.

Derogatis, L. R. (1994). Symptom Check List-90-R (SCL-90-R) administration, scoring and procedures manual (3. ed.). Baltimore, MD: National Computer Systems.

Dixon, J. B., Dixon, M. E., \& O`Brien, P. E. (2003). Depression in association with severe obesity: Changes with weight loss. Archives of Internal Medicine, 163(17), 2058-2065.

Dymek, M., Grange, D. L., Neven, K., \& Alverdy, J. (2002). Quality of life after Gastric Bypass Surgery: A cross-sectional study. Obesity Research, 10(11), 1135-1142.

Ellsworth, G. A., Strain, G. W., Strain, J. J., Vaillant, G. E., Knittle, J., \& Zumoff, B. (1986). Defense maturity ratings and sustained weight loss in obesity. Psychosomatics 27(11), 772-781.

Fabricatore, A. N., Wadden, T. A., Sarwer, D. B., \& Faith, M. S. (2005). Health-related quality of life and symptoms of depression in extremely obese persons seeking bariatric surgery. Obesity Surgery, 15(3), 304-309.

Franques, A. R. M., \& Ascencio, R. F. R. (2006). Depressão e obesidade. In A. R. M. Franques \& M. S. A. Loli (Eds.), Contribuições da psicologia na cirurgia da obesidade (pp. 125135). São Paulo, SP: Vetor.

Garrido, A. B., Jr. (2001). Cirurgia da obesidade mórbida: Como estamos [Versão eletrônica]. Revista Abeso, 2(4). Retrieved from http://www.abeso.org.br

Geloneze, B., \& Pareja, J. C. (2006). Cirurgia bariátrica cura a síndrome metabólica? Arquivos Brasileiros de Endocrinologia \& Metabologia, 50(2), 400-407.

Guimarães, L. P M., \& Yoshida, E. M. P. (2008). Doença de Crohn e Retocolite Ulcerativa Inespecífica: Alexitimia e Adaptação. Manuscrito submetido a publicação.

Guisado, M. J. A., Vaz, F. J., Alarcón, J., Béjar, A., Casado, M. J., \& Rubio, M. A. (2002). Psicopatologia en pacientes con obesidad mórbida poscirugía gástrica. Revista Cubana de Endocrinologia, 13(1), 29-34. 
Oliveira, J. H. A. \& Yoshida, E. M. P. (2009). Avaliação Psicológica de Obesos Grau III Antes e Depois de Cirurgia Bariátrica.

Jiriki-Babb, P., \& Geliebter, A. (2003). Comparison of psychological characteristics of binging and nonbinging obese, female outpatients. Eating Weight Disorder, 8(2), 173-177.

Kipper, L., Blaya, C., Teruchkin, B., Heldt, E., Isolan, L., Mezzomo, K., et al. (2005). Evaluation of defense mechanisms in adult with panic disorder: Before and after treatment. Journal of Nervous \& Mental Disease, 193(9), 619-624.

Kipper, L., Kipper, L., Blaya, C., Teruchkin, B., Heldt, E., Isolan, L., et al. (2004). Brazilian patients of panic disorder: The use of defense mechanisms and their association with severity. Journal of Nervous \& Mental Disease, 192(1), 58-64.

Laloni, D. T. (2001). Escala de avaliação de sintomas $-90-R$ - SCL-90-R: Adaptação, precisão e validade. Tese de Doutorado não-publicada, Pontifícia Universidade Católica de Campinas, SP.

Loli, M. S. A. (2000). Obesidade como sintoma: Uma leitura psicanalítica. São Paulo, SP: Vetor.

Maciel, M. J. N., \& Yoshida, E. M. P. (2006). Avaliação de alexitimia, neuroticismo e depressão em dependentes de álcool. Avaliação Psicológica, 5(1), 43-54.

Malone, M., \& Mayer-Alger, S. (2004). Binge status and quality of life after gastric bypass surgery: A one-year study. Obesity Research, 12(3), 473-481.

Mamplekou, E., Komesidou, V., Bissias, C., Papakonstantinou, A., \& Melissas, J. (2005). Psychological condition and quality of life in patients with morbid obesity before and after surgical weight loss. Obesity Surgery, 15(8), 1177-1184.

Marcus, D. A. (2004). Obesity and the impact of chronic pain. Clinical Journal of Pain, 20(3), 186-191.

Matos, M. I., Aranha, L. S., Faria, A. N., Ferreira, S. R. G., Bacaltchuck, J., \& Zanella, M. T. (2002). Binge eating disorder, anxiety, depression and body image in grade III obesity patients. Revista Brasileira de Psiquiatria, 24(4), 165-169.

Mello, C. A. P. (2001). Gastroplastia Vertical em Y de Roux: Resgate da qualidade de vida dos obesos. Dissertação de Mestrado não-publicada, Universidade Federal de Santa Catarina, Florianópolis, SC.

Monteiro, C. A., \& Conde, W. L. (2000). Tendência secular da desnutrição e da obesidade na infância na cidade de São Paulo (1974-1996). Revista de Saúde Pública (São Paulo), 34(Sup. 6), 52-61.

Morosin, A., \& Riva, G. (1997). Alexithymia in a clinical sample of obese women. Psychology Representative, 80(2), 387-394.

Oliveira, V. M., Linardi, R. C., \& Azevedo, A. P. (2004). Cirurgia bariátrica: Aspectos psicológicos e psiquiátricos. Revista de Psiquiatria Clínica, 31(4), 199-201.

Organização Pan-Americana da Saúde. (2003). Obesidade e excesso de peso. In Organização Pan-Americana da Saúde, Doenças crônico-degenerativas e obesidade: Estratégia mundial sobre alimentação saudável, atividade física e saúde (pp. 27). Brasília, DF: Autor.

Peixoto, G., \& Geloneze, B. (2006). Tratamento cirúrgico da obesidade no paciente diabético. In Sociedade Brasileira de Diabetes, Atualização Brasileira sobre Diabetes: Versão atualizada. Rio de Janeiro, RJ: Diagraphic.

Perry, J. C. (1990). Escala de Mecanismos de Defesa (D. Wiethaeuper \& E. M. P. Yoshida, Trads.). Cambridge, MA: Harvard Medical School.

Pinaquy, S., Chabrol, H., Simon, C., Louvet, J. P., \& Barbe, P. (2003). Emotion eating, alexithymia, and binge-eating disorder in obese women. Obesity Research, 11(2), 195-201.

Pregnolatto, A. P. F. (2005). Alexitimia e sintomas psicopatológicos em pacientes com insuficiência renal crônica. Dissertação de Mestrado não-publicada, Pontifícia Universidade Católica de Campinas, SP.
Regina, M. C. O. (2005). Alexitimia, ansiedade e depressão em portadores de glaucoma. Dissertação de Mestrado nãopublicada, Pontifícia Universidade Católica de Campinas, SP.

Romero, C. E. M., \& Zanesco, A. (2006). O papel dos hormônios leptina e grelina na gênese da obesidade. Revista de Nutrição, 19(1), 85-91.

Sánchez, R. S., López, A. J. C., Vargas, M. A., Téllez, Z. J. F., Vásquez, V. V., Arcila, M. D., et al. (2003). Prevalencia de trastornos psiquiátricos en pacientes con obesidad extrema candidatos a cirugía bariátrica. Revista de Investigación Clínica, 55(4), 400-406.

Sifneos, P. E. (1973). The prevalence of alexithymic characteristics in psychosomatic patients. Psychoterapy and Psychosomatics, 22, 255-262.

Silva, F. R. C. S. (2004). Validade simultânea da escala de avaliação de sintomas - 40/EAS-40 com estudantes universitários. Campinas, SP: Pontifícia Universidade Católica de Campinas. Relatório de pesquisa não-publicado.

Taylor, G. J., \& Bagby, R. M. (2004). New trends in alexithymia research. Psychotherapy and_Psychosomatics, 73, 68-77.

Taylor, G. J., Bagby, R. M., Ryan, D. P., Parker, J. D., Doody, K. F., \& Keefe, P. (1988). Criterion validity of the Toronto Alexithymia Scale. Psychosomatic Medicine, 50, 500-509.

Taylor, G. J., Ryan, D., \& Bagby, R. M. (1985). Toward the development of a new self-report alethymia scale. Psychotherapy and Psychosomatics, 44, 191-199.

Troisi, A., Scucchi, S., San Martino, L., Montera, P., D’Amore, A., \& Moles, A. (2001). Age specificity of the relationship between serum cholesterol and mood in obese women. Physiology Behavior, 72(3), 409-413.

Vasques, F., Martins, F. C., \& Azevedo, A. P. (2004). Aspectos psiquiátricos do tratamento da obesidade. Revista de Psiquiatria Clínica, 31(4), 195-198.

Viuniski, N. (2003). O manejo da obesidade infantil [Versão eletrônica]. Revista Abeso, 4(14). Retrieved from http:// www.abeso.org.br

Yoshida, E. M. P. (2000). Toronto Alexithymia Scale - TAS: Precisão e validade da versão em português. Psicologia: Teoria e Prática, 2(1), 59-74.

Yoshida, E. M. P. (2006). Avaliação de Alexitimia. Trabalho apresentado no II Congresso Brasileiro de Psicologia Ciência e Profissão, São Paulo, SP.

Yoshida, E. M. P. (2007). Validade da versão em português da Toronto Alexithymia Scale - TAS em amostra clínica. Psicologia: Reflexão e Crítica, 20(3), 389-396.

Yoshida, E. M. P., \& Silva, F. R. C. S. (2007). Escala de Avaliação de Sintomas - 40 (EAS-40): Validade e precisão em amostra não-clínica. Psicologia Escolar e Educacional, 11(1), 89-99.

Wagner, T. M. C. (2000). Obesidade na adolescência, imagem corporal, alexitimia e ansiedade. Tese de Doutorado nãopublicada, Universidade Federal do Rio Grande do Sul, Porto Alegre, RS.
Recebido: $27 / 12 / 2006$ $1^{a}$ revisão: $27 / 12 / 2007$ $2^{a}$ revisão: $24 / 05 / 2008$ $3^{a}$ revisão: $15 / 06 / 2008$ Aceite final: 23/06/2008 